\title{
COMPARAÇÃO ENTRE OS MÉTODOS DE FUMIGAÇÃO- EXTRAÇÃO E FUMIGAÇÃO-INCUBAÇÃO PARA DETERMINAÇÃO DO CARBONO DA BIOMASSA MICROBIANA EM UM LATOSSOLO ${ }^{(1)}$
}

\author{
Osvaldino Brandão-Junior ${ }^{(2)}$, Mariangela Hungria ${ }^{(3)}$, Julio Cezar \\ Franchini $^{(3)} \&$ Carlos Roberto Espindola ${ }^{(2)}$
}

\begin{abstract}
RESUMO
Um número crescente de trabalhos, inclusive no Brasil, vem sugerindo a avaliação do $\mathrm{C}$ da biomassa microbiana (CBM) como um bioindicador da qualidade dos solos. A maioria dos estudos emprega dois métodos: de fumigação-incubação (FI), pioneiro, em que o CBM é estimado pela diferença entre as quantidades de $\mathrm{CO}_{2}$ liberadas durante a incubação (10 dias) pelo solo fumigado e pelo solo nãofumigado, enquanto no de fumigação-extração (FE) essa determinação é feita a partir da extração e quantificação do $\mathrm{C}$ das amostras fumigadas e não-fumigadas após $24 \mathrm{~h}$. A correlação entre os dois métodos, porém, foi realizada em apenas três agroecossistemas brasileiros: na Amazônia, nos Cerrados e em três solos do Rio de Janeiro. Neste trabalho, os métodos FE e FI foram comparados em um Latossolo Vermelho distroférrico eutrófico típico do norte do Paraná. As avaliações foram feitas em amostras de solo de um ensaio realizado por 12 anos em Londrina, com seis tratamentos, incluindo três sistemas de manejo do solo (sistema plantio direto, PD; sistema preparo convencional com aração e gradagem, PC; e PD com uso de cruzador a cada três anos, $\mathrm{Cr}$ ) e dois sistemas de manejo de culturas em sucessão ou rotação. Foram realizadas coletas de solo, da camada de 0-10 cm, em quatro
\end{abstract}

\footnotetext{
(1) Recebido para publicação em março de 2006 e aprovado em agosto de 2008.

${ }^{(2)}$ Doutorando na Faculdade de Engenharia Agrícola, Universidade Estadual de Campinas - UNICAMP. Caixa Postal 6011, CEP 13083-875, Campinas, SP. E-mails: dinoagr@yahoo.com.br; cresp21@hotmail.com

(3) Pesquisadores da Embrapa Soja. Caixa. Postal 231, CEP 86001-970 Londrina (PR). E-mails: hungria@cnpso.embrapa.br; franchin@cnpso.embrapa.br
} 
fases do sistema de produção: pós-colheita de inverno (trigo na sucessão e tremoço na rotação); pós-aração de verão (dos tratamentos $\mathrm{PC}$ e Cr); pós-plantio de verão (soja na sucessão e milho na rotação); e na época de florescimento de verão. Em geral, não foi observada grande variabilidade em nenhum dos métodos, sendo o coeficiente de variação maior quanto menor o teor de CBM. Quando as coletas nas diferentes fases foram consideradas em conjunto, não houve diferença entre os métodos FE e FI em nenhum dos seis tratamentos estudados, bem como na análise conjunta de todos os tratamentos. Considerando todos os tratamentos, foi constatada correlação positiva significativa entre os métodos FE e FI, no entanto, na análise de cada tratamento, a significância ocorreu apenas para os tratamentos sob PD e PC em rotação ou sucessão de culturas, mas não para o uso do cruzador (Cr). Os resultados indicam que ambos os métodos podem ser utilizados para avaliar o CBM em condições semelhantes às do norte do Paraná, mas apontam a necessidade de se desenvolverem mais estudos comparativos entre os dois métodos nos solos brasileiros, em função de variações na quantidade e qualidade de matéria orgânica, assim como na intensidade de revolvimento do solo e incorporação de resíduos.

Termos de indexação: matéria orgânica, metodologia, plantio convencional, plantio direto, rotação de culturas.

\section{SUMMARY: COMPARISON OF THE METHODS FUMIGATION-EXTRACTION AND FUMIGATION-INCUBATION IN THE EVALUATION OF MICROBIAL BIOMASS C IN A DARK RED OXISOL}

More and more studies, including in Brazil, have suggested the use of microbial biomass carbon $(M B C)$ as a bioindicator of soil quality. Most studies employ two methods: the pioneer of fumigation-incubation (FI), in which the $\mathrm{MBC}$ is estimated based on $\mathrm{CO}_{2}$ emission from fumigated and non-fumigated soil samples after 10 days of incubation and fumigationextraction (FE), in which $M B C$ is estimated based on $C$ extraction from fumigated and nonfumigated soil samples after $12 \mathrm{~h}$. However, in Brazil the correlation between both methods has been evaluated in three ecosystems only: in the Amazon, the Cerrado and three soils of the State of Rio de Janeiro. In this paper the methods FE and FI were compared in a typical Dark Red Oxisol in the North of Paraná State. The analyses were performed in a 12 yearfield trial in Londrina with six treatments, including three soil managements (no-tillage, NT; conventional tillage with traditional ploughing and disking, CT; and NT using a field cultivator every three years, $F C$ ) and two crop management types (crop rotation, CR; or multiple cropping, MC) systems. Four soil samples $(0-10 \mathrm{~cm})$ were taken: after winter harvest (wheat in $C R$ and lupin in $M C$ ), after summer ploughing (for CT and FC treatments), after summer sowing (soybean in $C R$ and maize in $M C$ ) and in the summer crop flowering stage. In general the variability was low by both methods and the coefficient of variation was higher, the lower the MBC values. No differences between the FE and FI methods were detected when the four samplings at different stages as well as when all treatments were considered together. When all treatments were analyzed together, the correlation between FE and FI was positive and statistically significant. However, when each treatment was considered separately significance was observed for the treatments NT and CT in CR and $M C$, but not for FC. Results indicate that both methods may be used to evaluate $M B C$ under similar conditions in Northern Paraná, however, they also indicate that more studies should be performed in Brazilian soils comparing both methods as a function of qualitative and quantitative changes in the organic matter contents as well as in the incorporation of crop residues.

Index terms: organic matter, methodology, conventional tillage, no-tillage, crop rotation. 


\section{INTRODUÇÃO}

A biomassa microbiana do solo é definida como o componente microbiano vivo do solo, composto de bactérias (incluindo actinomicetos), fungos, microfauna e algas. Representa, em média, de 2 a $5 \%$ do $\mathrm{C}$ (Jenkinson \& Ladd, 1981) e de 1 a $5 \%$ do $\mathrm{N}$ total (Smith \& Paul, 1990) do solo. O conceito de que, para determinados estudos, todas as populações microbianas poderiam ser consideradas como um todo foi inicialmente proposto por Jenkinson (1966). Desde então, o papel relevante da biomassa microbiana do solo como componente crítico de vários ecossistemas naturais ou manipulados pelo homem tem sido confirmado, pois, entre outras funções, é o agente regulador da taxa de decomposição da matéria orgânica e da ciclagem dos elementos (Jenkinson \& Ladd, 1981), atuando, portanto, como fonte e dreno dos nutrientes necessários ao crescimento das plantas (Ladd et al., 1985).

Os trabalhos envolvendo a análise da biomassa microbiana do solo podem fornecer informações úteis sobre a dinâmica do reservatório lábil da matéria orgânica do solo, bem como indicar alterações decorrentes de diferentes práticas agrícolas, como o manejo dos solos e das culturas (Cattelan \& Vidor, 1990; Balota et al., 1998; Franchini et al., 2007) e o uso de fertilizantes orgânicos ou químicos e biocidas em geral (Brookes, 1995; Jordan et al., 1995). A biomassa microbiana tem sido sugerida, ainda, como um bioindicador da qualidade dos solos, uma vez que alguns estudos já demonstraram que essa avaliação é mais sensível a perturbações do solo do que as características químicas e físicas (Powlson et al., 1987; Brookes, 1995; Jordan et al., 1995; Balota et al., 1998; Franchini et al., 2007).

A biomassa microbiana do solo pode ser determinada por diversos métodos, como a respiração induzida por substrato, a determinação do conteúdo de ATP, microcalorimetria, contagem de células por microscopia direta (Anderson \& Domsch, 1978; Grisi \& Gray, 1986; Wardle, 1997). Contudo, os métodos mais utilizados são os de fumigação-incubação (Jenkinson \& Powlson, 1976a,b) e fumigação-extração (Vance et al., 1987a). Ambos os métodos são baseados na esterilização parcial (fumigação) de amostras de solos com clorofórmio, que apresenta as vantagens de amplo espectro, matando quase todas as populações microbianas em ação rápida, rompendo a membrana celular e liberando os constituintes internos, além de causar pouca alteração na composição da matéria orgânica do solo e de ser facilmente removido do solo (Jenkinson \& Powlson, 1976a; Wardle \& Parkinson, 1990b). No método FI, a biomassa microbiana é estimada com base no fluxo de $\mathrm{CO}_{2}$ liberado após a incubação de amostras de solo fumigadas e nãofumigadas por 10 dias (Jenkinson \& Powlson, 1976a,b). No método FE, por sua vez, a estimativa é feita a partir da extração e quantificação do $\mathrm{C}$ das amostras fumigadas e não-fumigadas após $24 \mathrm{~h}$ (Vance et al., 1987a).
Pelo pioneirismo e validação em vários estudos, o método FI tem sido utilizado como padrão para calibração de outros métodos de quantificação do $\mathrm{C}$ da biomassa microbiana (CBM). Desde a proposta de modificação do método FI por Vance et al. (1987a), visando facilitar as análises no laboratório, a eficiência dos dois métodos foi comparada em alguns estudos, a maioria conduzida em solos ácidos da Austrália e Nova Zelândia, e, em geral, correlações razoáveis foram obtidas (Sparling \& Zhu, 1993; Wardle \& Ghani, 1995). Em quase duas décadas de pesquisa foi possível constatar, também, que ambos os métodos apresentam vantagens e restrições. Entre as vantagens do FI, destaca-se a estimativa direta, com grande facilidade de execução, e o fornecimento da taxa de respiração microbiana (liberação de $\mathrm{CO}_{2}$ ) (Pfenning et al., 1992; Oliveira et al., 2001). Contudo, o FI apresenta restrições em solos ácidos (3,6 a 5,0) (Jenkinson \& Powlson, 1976a; Vance et al., 1987b,c), ou que receberam adições recentes de resíduos orgânicos (Sampaio et al., 1986). Pode haver dificuldade na escolha do controle que melhor expresse o nível de respiração basal do solo (Franzluebbers et al., 1999) e variações no $\mathrm{K}_{\mathrm{C}}$ (coeficiente do $\mathrm{C}$ microbiano convertido em $\mathrm{CO}_{2}$ após a fumigação e a incubação) com o tipo (Vance et al., 1987b) e pH do solo (Vance et al., 1987b,c), bem como com alterações na umidade do solo e com o esgotamento do substrato (Wardle \& Parkinson, 1990a). Já o método FE apresenta como principal vantagem de que não há dependência do estado fisiológico da população microbiana do solo (Vance et al., 1987a), não sendo necessário um controle apropriado (Sparling \& Zhu, 1993). Resultados mais confiáveis são obtidos em solos ácidos e na presença de substratos orgânicos frescos (Vance et al., 1987a), e o método permite a quantificação de outros nutrientes importantes da biomassa microbiana no mesmo extrato de solo, como o $\mathrm{P}$, o S e, principalmente, o N (Brookes et al., 1985). Quanto às desvantagens do método $\mathrm{FE}$, verifica-se que, na ausência de um analisador automático de $\mathrm{C}$, o método requer a utilização de dicromato de $\mathrm{K}$, que é tóxico, e em solos com diferentes teores de argila e matéria orgânica, o coeficiente $\mathrm{K}_{\mathrm{CE}}$ (coeficiente de extração do $\mathrm{C}$ microbiano após a fumigação e extração) empregado pode apresentar maior variabilidade do que o $\mathrm{K}_{\mathrm{C}}$ do $\mathrm{FI}$ (Jenkinson, 1988).

No Brasil, um número crescente de trabalhos tem avaliado o CBM por FI ou FE, contudo poucos estudos compararam os dois métodos nos solos brasileiros. Na Amazônia, Pfenning et al. (1992), Feighl et al. (1995) e Geraldes et al. (1995) verificaram boas correlações entre os dois métodos mesmo em solos ácidos, porém o método FE mostrou-se mais adequado, por apresentar menor variabilidade nas análises. Rodrigues et al. (1994) fizeram essa comparação em três tipos de solos no Rio de Janeiro, enquanto Oliveira et al. (2001) utilizaram um solo típico da região dos Cerrados sob vegetação nativa ou sob cultivo. Em ambos os estudos, as correlações obtidas entre os dois 
métodos foram razoáveis, contudo Rodrigues et al. (1994) constataram maior exatidão do FE com o decréscimo da percentagem de $\mathrm{C}$ total do solo.

O norte do Paraná vem se destacando por pesquisas e tecnologias na área de conservação dos solos, com os ensaios pioneiros e o primeiro estabelecimento de lavoura em sistema plantio direto no início da década de 1970 (FEBRAPDP, 2006). São também do Paraná alguns trabalhos sugerindo a utilização do CBM como bioindicador de qualidade dos solos (Balota et al., 1998; Franchini et al., 2007). Contudo, a eficácia dos dois métodos de avaliação do CBM ainda não foi validada para os solos típicos do norte do Estado. O objetivo deste trabalho foi comparar os métodos FI e FE em um ensaio realizado há 12 anos em Londrina, PR, em um Latossolo Vermelho distroférrico eutrófico, sob diferentes sistemas de manejo do solo e das culturas.

\section{MATERIAL E MÉTODOS}

\section{Ensaio experimental}

As avaliações foram realizadas em um ensaio estabelecido na safra 1988/1989 na Estação Experimental da Embrapa Soja (23 ${ }^{\circ} 11$ ' S, $51^{\circ} 11^{\prime} \mathrm{W}, 585 \mathrm{~m}$ de altitude), em Londrina, norte do Paraná, em um Latossolo Vermelho distroférrico eutrófico ou Rhodic Eutrudox (classificação norteamericana). O clima em Londrina é classificado como subtropical úmido, $C$ fa, de acordo com a classificação de Köppen.

O experimento consistiu de diferentes manejos de solo envolvendo aração e gradagem e diferentes sistemas de culturas, com parcelas de $15 \mathrm{~m}$ de comprimento por $8 \mathrm{~m}$ de largura. O solo é caracterizado por um teor elevado de argila $\left(710 \mathrm{~g} \mathrm{~kg}^{-1}\right.$ de argila; $82 \mathrm{~g} \mathrm{~kg}^{-1}$ de silte; $208 \mathrm{~g} \mathrm{~kg}^{-1}$ de areia) e bons níveis de fertilidade na camada de $0-10 \mathrm{~cm}: \mathrm{pH} 5,3$ a 5,5, teores de $\mathrm{N}$ de 1,9 a 2,4 $\mathrm{g} \mathrm{dm}^{-3}$ e de $\mathrm{C}$ de 18,1 a 22,5 $\mathrm{g} \mathrm{dm}^{-3}$. $\mathrm{O}$ delineamento experimental do ensaio foi em blocos ao acaso com quatro repetições, com os tratamentos em esquema fatorial, incluindo três sistemas de preparo do solo (sistema plantio direto, $\mathrm{PD}$; sistema preparo convencional com aração e gradagem, PC; e PD com uso de cruzador a cada três anos, $\mathrm{Cr}$ ) e dois sistemas de culturas em sucessão [S, com soja (Glycine max) e trigo (Triticum aestivum)] e rotação [R, com tremoço (Lupinus spp.)/milho (Zea mays)/aveia-preta (Avena strigosa)/soja/trigo/soja/trigo/soja], totalizando seis combinações de tratamentos. Nas safras de verão de 1998/1999, 1999/2000, 2000/2001, as parcelas foram cultivadas com soja, e nas de inverno de 1999 e 2000 , com trigo. No inverno de 2001 , as parcelas na rotação foram cultivadas com tremoço e aquelas na sucessão com trigo, e no verão de 2001/2002, foram cultivados milho nas de rotação e soja nas de sucessão. O cruzador foi utilizado em outubro após a colheita do trigo. As coletas foram realizadas a partir da colheita do trigo e da rolagem com rolo-faca do tremoço em setembro de 2001 até o florescimento da soja e pendoamento do milho no verão de 2001/2002.

\section{Coleta das amostras}

As coletas do solo $(0-10 \mathrm{~cm})$ foram realizadas sempre pela manhã, nos períodos pós-colheita de inverno (setembro/2001), pós-aração de verão (outubro/ 2001), pós-plantio de verão (novembro/2001) e na época de florescimento de verão (janeiro/2002).

De cada uma das parcelas, foram coletadas seis subamostras de solo deformadas, da camada de 0-10 cm, nas entrelinhas, com uma pá reta. As subamostras foram homogeneizadas, peneiradas ( $4 \mathrm{~mm}-5 \mathrm{mesh}$ ), armazenadas em saco plástico, dando origem a uma amostra composta com aproximadamente $1 \mathrm{~kg}$ de solo. No campo, as amostras foram mantidas em caixas de isopor com gelo até o transporte para o laboratório, onde foram mantidas sob refrigeração até o dia seguinte, quando se iniciaram os procedimentos específicos de cada método de determinação da biomassa microbiana.

\section{Análise do C da biomassa microbiana}

\section{Método de fumigação-incubação (FI)}

O método utilizado foi adaptado de Jenkinson \& Powlson (1976a,b). Em frascos de $300 \mathrm{~mL}$, foram pesadas quatro replicatas de cada amostra composta de solo, cada uma com $100 \mathrm{~g}$. Duas replicatas foram fumigadas e incubadas, e as outras duas foram incubadas sem fumigação.

Os frascos contendo as amostras a serem fumigadas foram colocados em dessecadores ( 8 a 10 frascos por dessecador) contendo uma placa de Petri com $50 \mathrm{~mL}$ de clorofórmio isento de álcool. Os dessecadores, após terem a parede interna recoberta com papel-toalha umedecido, foram fechados com a utilização de vaselina e submetidos a vácuo por 5 10 min e deixados em repouso em temperatura ambiente e no escuro por $24 \mathrm{~h}$. Após esse período, os dessecadores foram abertos para aeração e eliminação do clorofórmio. Para a retirada do clorofórmio, os dessecadores foram evacuados com bomba de ar por cerca de $3 \mathrm{~min}$, procedimento repetido por cinco a seis vezes. Os frascos contendo as amostras não-fumigadas também foram deixados em repouso no escuro por $24 \mathrm{~h}$ em temperatura ambiente.

As umidades de todas as amostras fumigadas e não-fumigadas foram corrigidas para $24 \%$ da capacidade de campo, com adição de água deionizada. Às amostras fumigadas foi adicionado $1 \mathrm{~g}$ do solo da mesma amostra não-fumigada (reinoculação), seguindo-se a homogeneização delas.

A seguir, realizou-se a incubação das amostras em potes de vidro de $2 \mathrm{~L}$, onde foram colocados também um frasco de $100 \mathrm{~mL}$ contendo $50 \mathrm{~mL}$ de $\mathrm{NaOH}$ $0,5 \mathrm{~mol} \mathrm{~L}^{-1}$ e um pote plástico (embalagem de filme fotográfico) com $20 \mathrm{~mL}$ de água. Adicionou-se vaselina 
à borracha de vedação dos frascos antes de fechá-los. Os potes foram incubados no escuro, a $25^{\circ} \mathrm{C}$, por 10 dias. Para cada repetição, foi incubado um frasco sem solo (branco), mas com $\mathrm{NaOH} 0,5 \mathrm{~mol} \mathrm{~L}^{-1}$ e água deionizada.

Ao final do período de incubação, o $\mathrm{NaOH}$ dos frascos dos potes das amostras fumigadas e nãofumigadas foi titulado com $\mathrm{HCl} \mathrm{0,2} \mathrm{mol} \mathrm{L}^{-1}$. Para isso, em alíquotas contendo $5 \mathrm{~mL}$ do $\mathrm{NaOH}$, adicionaramse $1 \mathrm{~mL}$ de solução saturada de $\mathrm{BaCl}_{2}$, para retirada de carbonato do meio $\left(\mathrm{Na}_{2} \mathrm{CO}_{3}\right), 20 \mathrm{~mL}$ de água deionizada e três gotas do indicador (fenolftaleína), mantendo a solução agitada por meio de agitador magnético. As amostras em branco também foram tituladas.

Após a titulação do $\mathrm{NaOH}$, calculou-se a quantidade de $\mathrm{C}-\mathrm{CO}_{2}$ liberada das amostras fumigadas e nãofumigadas. O CBM foi calculado a partir da expressão:

$$
\mathrm{CBM}=(\mathrm{C} \text { fumigado }-\mathrm{C} \text { não-fumigado }) / \mathrm{K}_{\mathrm{C}}
$$

Para o $\mathrm{K}_{\mathrm{C}}$ assume-se que $41 \%$ do CBM do solo foi convertido em $\mathrm{CO}_{2}$ durante os 10 dias de incubação após a fumigação (Anderson \& Domsch, 1978). É importante salientar que os resultados de Sampaio et al. (1986) sugerem que o $\mathrm{K}_{\mathrm{C}}=0,41$ poderia ser utilizado de modo generalizado também para solos tropicais. Os resultados foram expressos após a correção da umidade do solo.

\section{Método de fumigação-extração (FE)}

O método utilizado foi adaptado de Vance et al. (1987a). Foram pesadas quatro replicatas de $20 \mathrm{~g}$ de cada amostra de solo, em frascos de $300 \mathrm{~mL}$, sendo duas fumigadas e duas não. O processo de fumigação das amostras foi o mesmo descrito no item anterior. A seguir, as amostras foram colocadas em repouso no escuro por $24 \mathrm{~h}$ em temperatura ambiente.

Após esse período, as amostras foram submetidas ao processo de extração do $\mathrm{C}$ por meio da adição de $50 \mathrm{~mL}$ de solução extratora $\left(\mathrm{K}_{2} \mathrm{SO}_{4} 0,5 \mathrm{~mol} \mathrm{~L}^{-1}\right)$, submetidas à agitação de 170-175 rpm por uma hora, seguida de centrifugação por $10 \mathrm{~min}$ a $3.000 \mathrm{rpm}$, e filtradas em papel qualitativo para análise do C.

$\mathrm{O}$ teor de $\mathrm{C}$ nos extratos das amostras foi determinado por meio da oxidação do C com a utilização de permanganato e pirofosfato, segundo Bartlett \& Ross (1988). Dos extratos filtrados, foram retiradas alíquotas de $0,5 \mathrm{~mL}$, transferidas para tubos de ensaio, adicionando-se $0,5 \mathrm{~mL}$ da solução extratora $\left(\mathrm{K}_{2} \mathrm{SO}_{4}\right.$ 0,5 $\mathrm{mol} \mathrm{L}^{-1}$ ), $1 \mathrm{~mL}$ da solução de Mn (III) - pirofosfato e $1 \mathrm{~mL}$ de $\mathrm{H}_{2} \mathrm{SO}_{4}$ concentrado. Preparou-se uma curva-padrão de $0\left(\mathrm{~K}_{2} \mathrm{SO}_{4} 0,5 \mathrm{~mol} \mathrm{~L}{ }^{-1}\right)$ a $120 \mathrm{mg} \mathrm{L}^{-1}$ de C, utilizando-se ácido oxálico como padrão; pipetaram-se $1 \mathrm{~mL}$ de cada padrão, $1 \mathrm{~mL}$ de Mn (III) - pirofosfato e $1 \mathrm{~mL}$ de $\mathrm{H}_{2} \mathrm{SO}_{4}$ concentrado. Homogeneizou-se o conjunto por agitação, deixando-o em repouso por, no mínimo, 16 h e, depois, efetuou-se a leitura da absorbância a $495 \mathrm{~nm}$ em espectrofotômetro digital B342 (Micronal).

O CBM foi estimado pela diferença de $\mathrm{C}$ entre as amostras e calculado a partir da expressão:

$$
\mathrm{CBM}=(\mathrm{C} \text { fumigado }-\mathrm{C} \text { não-fumigado }) / \mathrm{K}_{\mathrm{CE}}
$$

Embora o $\mathrm{K}_{\mathrm{CE}}$ utilizado por Vance et al. (1987a,b) seja de 0,38 , foi utilizado o valor do $\mathrm{K}_{\mathrm{C}}$ de 0,41 para evitar valores superestimados pelo método $\mathrm{FE}$, hipótese levantada em alguns estudos (Feigl et al., 1995; Oliveira et al., 2001).

\section{Análises estatísticas}

Para as análises estatísticas, foi utilizado o programa Statistical Analysis System (SAS) versão 8.2 (SAS, 2001). Para se verificar a diferença entre o CBM obtido nas diferentes coletas, tanto pelo método FI como pelo FE, realizou-se o teste de ANOVA para comparar as quatro coletas entre si e, no caso de diferença significativa, realizou-se o teste de Tukey para determinar entre quais coletas houve essa diferença. Para determinar se houve diferença entre os métodos FE e FI, aplicou-se o teste $t$ não-pareado para as médias das quatro coletas, considerando-se os diferentes sistemas de manejo do solo e de cultura utilizados. O teste de correlação linear foi aplicado para se verificar a correlação entre o CBM e os coeficientes de variação obtidos por FE e FI, bem como do CBM obtido pelos dois métodos. O teste de correlação entre o CBM obtido nos dois métodos foi realizado de duas maneiras: considerando separadamente os diferentes sistemas de manejo e de culturas e, depois, apenas os dois métodos, FE e FI. Como informação complementar, o teste de ANOVA foi utilizado para comparar os sistemas de manejo e culturas em cada método; no caso de diferença significativa, realizou-se o teste de Tukey para determinar entre quais sistemas houve essa diferença.

\section{RESULTADOS E DISCUSSÃO}

Neste estudo, inicialmente procedeu-se à comparação dos valores de CBM obtidos por FE e por FI (Quadro 1) nos seis tratamentos e nas quatro coletas realizadas. $\mathrm{Na}$ primeira (pós-colheita de inverno) e na quarta (florescimento de verão) coletas, os maiores valores de CBM foram obtidos pelo método FE, em média 63 e 69 \% superiores aos valores obtidos por FI, respectivamente. Ao contrário, na segunda (pós-aração de verão) e na terceira (pós-plantio de verão) coletas, os valores de CBM obtidos por FI foram, em média, $67 \%$ superiores aos obtidos por FE. A variabilidade do método $\mathrm{FE}$, estimada pelo desviopadrão da média, foi inferior à do FI apenas na primeira coleta; nas demais foi equivalente em ambos os métodos (Quadro 1). É importante observar, também, que a variabilidade detectada neste estudo 
Quadro 1. Carbono da biomassa microbiana [valor médio ${ }^{(1)}$ e desvio-padrão (s)] em diferentes épocas de coleta, obtido pelos métodos da fumigação-extração e fumigação-incubação para diferentes sistemas de manejo do solo e das culturas; e resultado do teste ANOVA e Tukey com os níveis de probabilidade de significância $(p)$

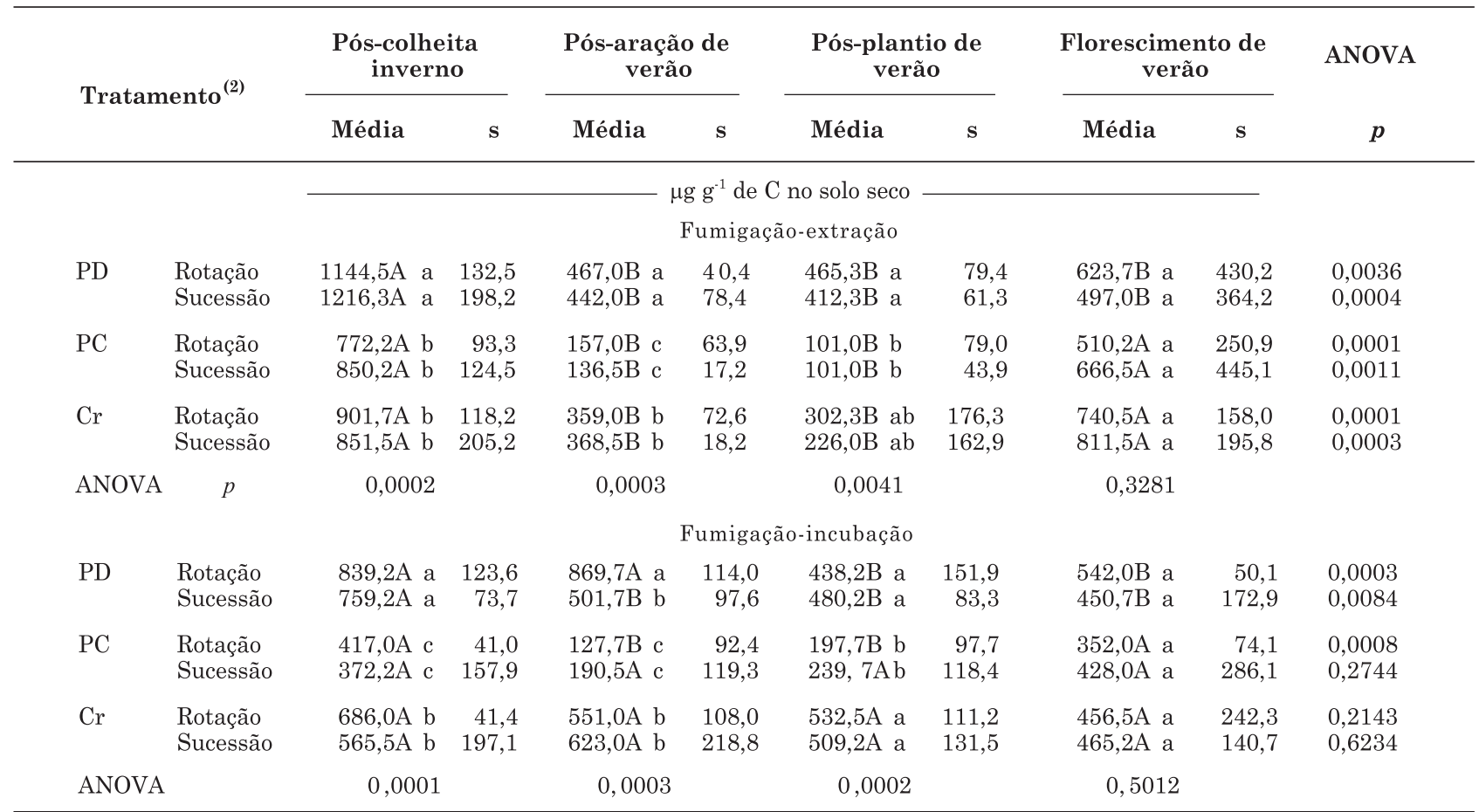

(1) Médias seguidas de mesma letra, maiúscula na linha e minúscula na coluna, não diferem estatisticamente pelo teste de Tukey, no nível de significância $(p)$ indicado. ${ }^{(2)}$ Sistema plantio direto (PD), plantio convencional (PC) e PD com uso de cruzador a cada três anos $(\mathrm{Cr})$ em rotação $(\mathrm{R})$ e sucessão de culturas $(\mathrm{S})$, conforme especificado no Material e Métodos.

foi semelhante àquela encontrada por Rodrigues et al. (1994) e superior à econtrada em estudo com solos da Amazônia (Pfenning et al., 1992).

Coeficientes de correlação negativos foram observados entre os valores de CBM e o coeficiente de variação (CV \%), contudo com significância estatística apenas para o método FI (Figura 1). Conseqüentemente, ambos os métodos devem ser utilizados com cautela em solos com baixos teores de CBM, pois a variabilidade tende a aumentar. Esses resultados são similares aos reportados por Rodrigues et al. (1994), obtidos em solos do Rio de Janeiro, onde os autores observaram que a exatidão de ambos os métodos diminuía com a redução dos teores de $\mathrm{C}$ total do solo, porém naquele trabalho os autores constataram maior exatidão com o método $\mathrm{FE}$, diferentemente do resultado obtido neste estudo.

Diversos estudos realizados no Brasil constataram diferenças no teor de CBM entre épocas de amostragem, principalmente em função do ciclo das plantas, da adição de resíduos vegetais, da pluviometria e da temperatura (Cattelan \& Vidor, 1990; Balota et al., 1998; Franchini et al., 2007). Neste estudo, ao se avaliar o efeito da época de amostragem no CBM, o método FE conseguiu detectar diferenças em todos os tratamentos, mas no método FI essas diferenças foram observadas em apenas três dos seis tratamentos (Quadro 1). Isso pode indicar maior sensibilidade do FE para detecção de diferenças.

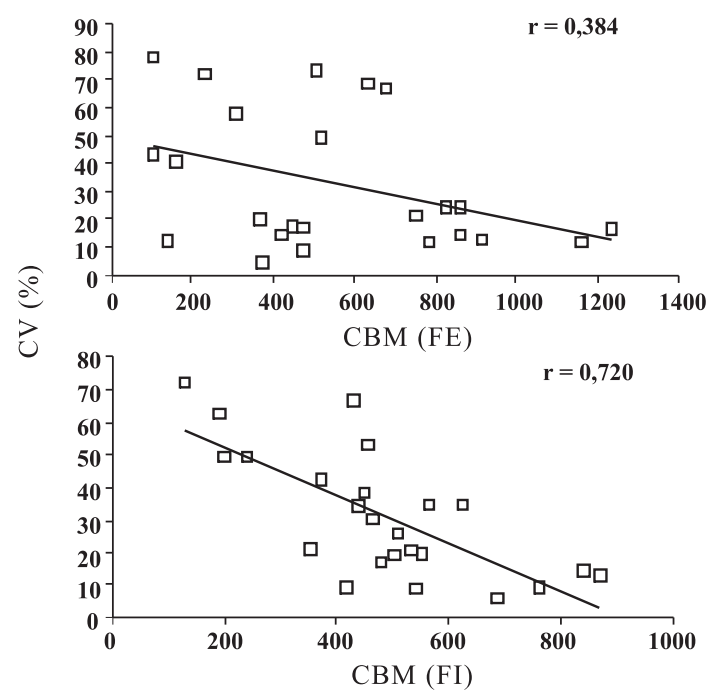

Figura 1. Correlação entre o coeficiente de variação (CV) e o carbono da biomassa microbiana (CBM) obtidos pelos métodos de (a) fumigaçãoincubação (F1) e (b) fumigação-extração (FE). 


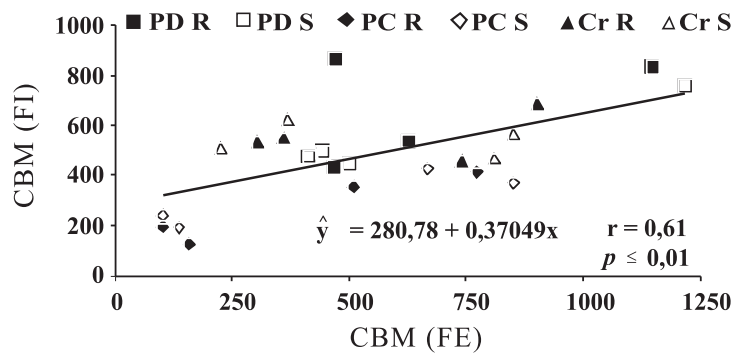

Figura 2. Correlação e coeficiente lineares entre o carbono da biomassa microbiana (CBM) determinados pelos métodos de fumigaçãoincubação (FI) e fumigação-extração (FE), em um ensaio conduzido em um Latossolo Vermelho distroférrico de Londrina, PR, sob diferentes manejos de solo e das culturas.
A aplicação do teste $t$ não-pareado para as médias das quatro coletas, considerando-se os diferentes sistemas de manejo do solo e de cultura, indicou que não houve diferença entre os métodos FE e FI em nenhum dos seis tratamentos estudados (Quadro 2). Além disso, quando todos os tratamentos foram considerados em conjunto, foi constatada uma correlação positiva e significativa entre o FI e o FE (Figura 2), validando o uso deste último para solos típicos do norte do Paraná. Contudo, considerando-se cada um dos tratamentos, coeficientes de correlação significativos entre os métodos FE e FI foram obtidos para os tratamentos sob PD e PC em rotação ou sucessão de culturas, mas não para o uso do cruzador (Quadro 3).

Os resultados deste trabalho encontram respaldo nos de Franchini et al. (2007), que constataram, nas

Quadro 2. Teor de carbono da biomassa microbiana pelos métodos de fumigação-extração e fumigaçãoincubação, em diferentes sistemas de manejo do solo e das culturas

\begin{tabular}{|c|c|c|c|c|c|c|}
\hline \multirow{2}{*}{\multicolumn{2}{|c|}{ Tratamento $^{(1)}$}} & \multicolumn{2}{|c|}{ F umigação-extração } & \multicolumn{2}{|c|}{ Fumigação-incubação } & \multirow{2}{*}{$\begin{array}{c}\text { Teste } t^{(3)} \\
p\end{array}$} \\
\hline & & Média & $\mathbf{s}$ & Média & $\mathbf{s}$ & \\
\hline $\mathrm{PD}$ & $\begin{array}{l}\text { Rotação } \\
\text { Sucessão }\end{array}$ & $\begin{array}{l}675,1^{(2)} \\
641,9\end{array}$ & $\begin{array}{l}353,4 \\
393,2\end{array}$ & $\begin{array}{l}672,2 \\
547,9\end{array}$ & $\begin{array}{l}218,5 \\
163,0\end{array}$ & $\begin{array}{l}0,9780 \\
0,3843\end{array}$ \\
\hline $\mathrm{PC}$ & $\begin{array}{l}\text { Rotação } \\
\text { Sucessão }\end{array}$ & $\begin{array}{l}385,1 \\
438,5\end{array}$ & $\begin{array}{l}309,7 \\
396,1\end{array}$ & $\begin{array}{l}273,6 \\
307,6\end{array}$ & $\begin{array}{l}139,2 \\
192,0\end{array}$ & $\begin{array}{l}0,1990 \\
0,2435\end{array}$ \\
\hline $\mathrm{Cr}$ & $\begin{array}{c}\text { Rotação } \\
\text { Sucessão }\end{array}$ & $\begin{array}{l}575,9 \\
564,4\end{array}$ & $\begin{array}{l}288,3 \\
317,0\end{array}$ & $\begin{array}{l}556,5 \\
540,8\end{array}$ & $\begin{array}{l}155,6 \\
168,9\end{array}$ & $\begin{array}{l}0,8140 \\
0,7944\end{array}$ \\
\hline
\end{tabular}

(1) Sistema plantio direto (PD), plantio convencional (PC) e PD com uso de cruzador a cada três anos (Cr) em rotação e sucessão de culturas. ${ }^{(2)}$ Os dados correspondem à média e ao desvio-padrão (s) de quatro épocas de amostragem. ${ }^{(3)}$ Teste $t$ não-pareado e níveis de probabilidade de significância $(p)$.

Quadro 3. Teor de carbono da biomassa microbiana (CBM), coeficiente linear (r) e significância da correlação (p) entre os métodos de fumigação-incubação (FI) e fumigação-extração (FE), em cada sistema de manejo do solo e de culturas. Os valores de $r$ e $p$ foram obtidos considerando-se as quatro repetições em cada época de coleta

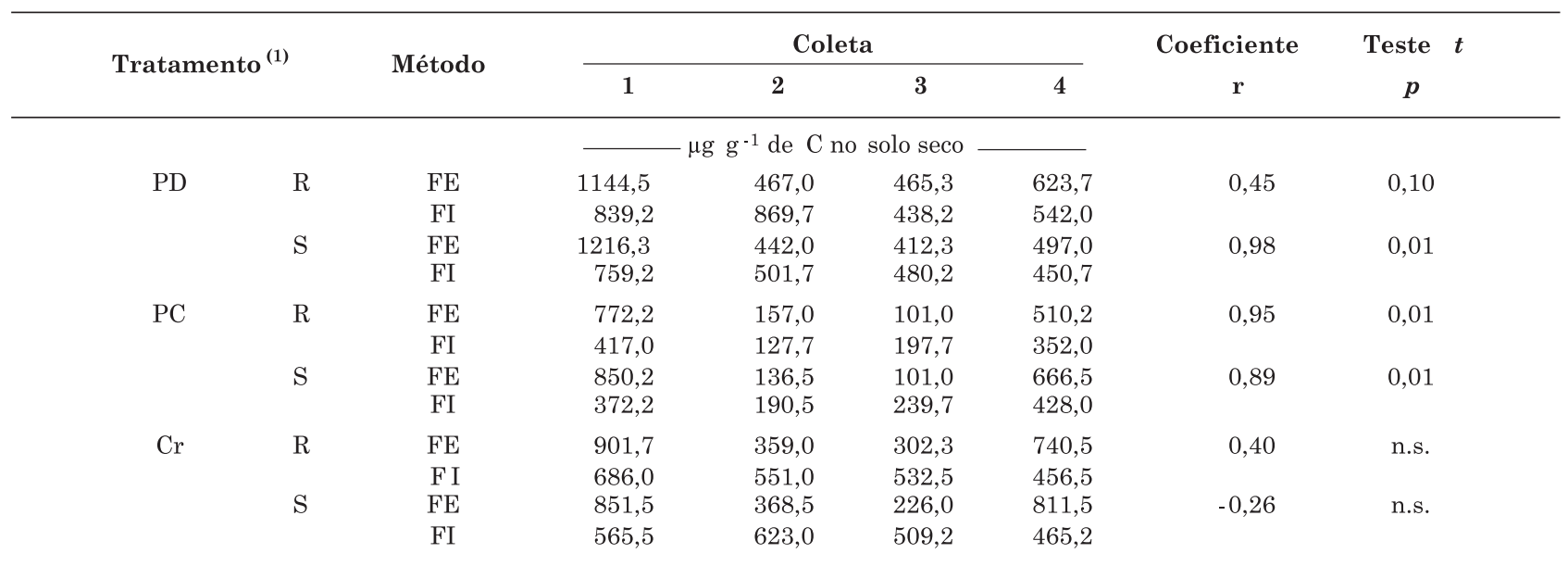

\footnotetext{
(1) Sistema plantio direto (PD), plantio convencional (PC) e PD com uso de cruzador a cada três anos (Cr) em rotação (R) e
} sucessão de culturas (S). 
mesmas condições de Londrina, que, em apenas cinco anos de PC, o teor de CBM do solo era bastante inferior ao do PD. Neste estudo, a aração e a gradagem no PC resultaram em decréscimo nos valores de CBM em relação ao $\mathrm{PD}$ (exceto na última coleta), estimados tanto pelo método FI quanto por FE. O uso do cruzador também resultou, em alguns casos, em decréscimo no CBM em comparação com os solos sem nenhum revolvimento (PD), sendo esse efeito significativo apenas na coleta pós-colheita de inverno em ambos os métodos e na coleta pós-aração de verão no método $\mathrm{FE}$ (Quadro 1). Contudo, neste estudo, a falta de correlação significativa entre os dois métodos de análise do CBM nos tratamentos com cruzador, em que houve revolvimento do solo e incorporação parcial dos resíduos, aponta para a necessidade de mais estudos comparativos, tanto no caso de incorporação de substratos frescos quanto no de matéria orgânica já estabilizada. Nos Cerrados, Oliveira et al. (2001) constataram que, em amostras sob cultivo, os valores de CBM obtidos pelo método FE foram, em geral, superiores aos estimados pelo método FI, o que nem sempre ocorreu em sistemas sob vegetação nativa. Os autores sugerem que é possível que cada um dos solos sob vegetação nativa possua um $\mathrm{K}_{\mathrm{EC}}$ diferenciado, em razão de diferenças na matéria orgânica e nas propriedades físicas, particularmente textura. Em conjunto com os dados deste ensaio, os resultados de estudos comparativos conduzidos no Brasil indicam que, nos solos brasileiros, a limitação dos métodos FI ou FE parece não estar na acidez do solo, e sim na qualidade e quantidade de matéria orgânica e de resíduos vegetais incorporados.

\section{AGRADECIMENTOS}

Ao Dr. Eleno Torres, responsável pelo ensaio na Embrapa Soja. A Rosinei A. Souza, Manoel P. da Silva e Luciano Souza, pelo apoio na condução das análises. Ao CNPq (Conselho Nacional de Desenvolvimento Científico e Tecnológico), pela concessão da bolsa de doutorado a O. Brandão-Junior. M. Hungria é bolsista de pesquisa do CNPq (300698/2007-0). Projeto financiado parcialmente pelo $\mathrm{MCT} / \mathrm{CNPq} / \mathrm{PRONEX} /$ Instituto do Milênio.

\section{LITERATURA CITADA}

ANDERSON, T.H. \& DOMSCH, K.H. A physiological method for the quantitative measurement of microbial biomass in soils. Soil Biol. Biochem., 10:215-221, 1978.

BALOTA, E.L.; COLOZZI-FILHO, A.; ANDRADE, D.S. \& HUNGRIA, M. Biomassa microbiana e sua atividade em solos sob diferentes sistemas de preparo e sucessão de culturas. R. Bras. Ci. Solo, 22:641-649, 1998.
BARTLETT, R.J. \& ROSS, D.N. Colorimetric determination of oxidizable carbon in acid soil solutions. Soil Sci. Soc. Am. J., 52:1191-1192, 1988.

BROOKES, P.C. The use microbial parameters in soil pollution by heavy metals. Biol. Fert. Soils, 19:269-279, 1995.

BROOKES, P.C.; LANDMAN, A.; PRUDEN, G. \& JENKINSON, D.S. Chloroform fumigation and the release of soil nitrogen: A rapid direct extraction method to measure microbial biomass nitrogen in soil. Soil Biol. Biochem., 17:837-842, 1985.

CATTELAN, A.J. \& VIDOR, C. Sistemas de culturas e a população microbiana do solo. R. Bras. Ci. Solo, 14:125132, 1990.

FEDERAÇÃO BRASILEIRA DE PLANTIO DIRETO NA PALHA-FEBRAPDP. Disponível em: <http:/ www.febrapdp.org.br/br/port/historico.html $>$ Acesso em 20 out de 2008.

FEIGL, B.J.; SPARLING, G.P.; ROSS, D.J. \& CERRI, C.C. Soil microbial biomass in Amazonian soils: Evaluation of methods and estimates of pool sizes. Soil Biol. Biochem., 27:1467-1472, 1995.

FRANCHINI, J.C.; CRISPINO, C.C.; SOUZA, R.A.; TORRES, E. \& HUNGRIA, M. Microbiological parameters as indicators of soil quality under various tillage and croprotation systems in Southern Brazil. Soil Till. Res., 92:18$29,2007$.

FRANZLUEBBERS, A.J.; HANEY, R.L. \& HONS, F.M. Relationships of chloroform fumigation-incubation to soil organic matter pools. Soil Biol. Biochem., 31:395-405, 1999.

GERALDES, A.P.A.; CERRI, C.C. \& FEIGL, B.J. Biomassa microbiana de solo sob pastagens na Amazônia. R. Bras. Ci. Solo, 19:55-60, 1995.

GRISI, B.M. \& GRAY, T.R.G. Comparação dos métodos de fumigação, taxa de respiração em resposta à adição de glicose e conteúdo de ATP para estimar a biomassa microbiana dos solos. R. Bras. Ci. Solo, 10:109-115, 1986.

JENKINSON, D.S. Determination of microbial biomass carbon and nitrogen in soils. In: WILSON, J.R., ed. Advances in nitrogen cycling in agricutural systems. Wallingford, $\mathrm{CAB}$ International, 1988. p.368-386.

JENKINSON, D.S. Studies on the decomposition of plant material in soil. II. Partial sterilisation of soil and the soil biomass. J. Soil Sci., 17:280-302, 1966.

JENKINSON, D.S. \& LADD, J.N. Microbial biomass in soils: Measurement and turnover. In: PAUL, E.A. \& LADD, J.N., eds. Soil biochemistry. New York, Marcel Decker, 1981. v.5. p.415-471.

JENKINSON, D.S. \& POWLSON, D.S. The effects of biocidal treatments on metabolism in soil. I. Fumigation with chloroform. Soil Biol. Biochem., 8:167-177, 1976a.

JENKINSON, D.S. \& POWLSON, D.S. The effect of biocidal treatments on metabolism in soil. V. A method of measuring soil biomass. Soil Biol. Biochem., 8:209-213, 1976b. 
JORDAN, D.; KREMER, R.J.; BERGFIELD, W.A.; KIM, K.Y. \& CACNIO-HUBBARD, V.N. Evaluation of microbial methods as potential indicators of soil quality in historical agricultural fields. Biol. Fert. Soils, 19:297-302, 1995.

LADD, J.N.; AMATO, M. \& OADES, J.M. Decomposition of plant material in Australian soils. III. Residual organic and microbial biomass $\mathrm{C}$ and $\mathrm{N}$ from isotope-labeled legume material and soil organic matter, decomposing under field conditions. Austr. J. Soil Res., 23:603-611, 1985.

OLIVEIRA, J.R.A.; MENDES, I.C. \& VIVALDI, L. Carbono da biomassa microbiana em solos de cerrado sob vegetação nativa e sob cultivo: Avaliação dos métodos fumigaçãoincubação e fumigação-extração. R. Bras. Ci. Solo, 25:863871, 2001.

PFENNING, L.; EDUARDO, B.P. \& CERRI, C.C. Os métodos da fumigação-incubação e fumigação-extração na estimativa da biomassa microbiana dos solos da Amazônia. R. Bras. Ci. Solo, 16:31-37, 1992.

POWLSON, D.S.; BROOKES, P.C. \& CHRISTENSEN, B.T Measurement of soil microbial biomass provides an early indication of changes in total soil organic matter due to straw incorporation. Soil Biol. Biochem., 19:159-164, 1987.

RODRIGUES, E.F.G.; GUERRA, J.G.M.; ALMEIDA, D.L. \& DE-POLLI, H. Biomassa microbiana de carbono de solos de Itaguaí (RJ): Comparação entre os métodos fumigaçãoincubação e fumigação-extração. R. Bras. Ci. Solo, 18:427432, 1994.

SAMPAIO, E.V.S.B.; SALCEDO, I.H. \& MAIA, L.C. Limitações no cálculo da biomassa microbiana determinada pelo método da fumigação em solos com adição recente de substrato orgânico $\left({ }^{14} \mathrm{C}\right)$. R. Bras. Ci. Solo, 10:31-35, 1986.

SAS Institute. SAS proprietary software release 8.2. Cary, 2001.
SMITH, J.L. \& PAUL, E.A. The significance of soil microbial biomass estimations. In: BOLLAG, J. \& STOTZKY, D.G., eds. Soil biochemistry. New York, M. Dekker, 1990. v.6. p.357-396.

SPARLING, G. \& ZHU, C. Evaluation and calibration of biochemical methods to measure microbial biomass $\mathrm{C}$ and $\mathrm{N}$ in soils from Western Australia. Soil Biol. Biochem., 25:1793-1801, 1993.

VANCE, E.D.; BROOKES, P.C. \& JENKINSON, D.S. An extraction method for measuring soil microbial biomass C. Soil Biol. Biochem., 19:703-707, 1987a.

VANCE, E.D.; BROOKES, P.C. \& JENKINSON, D.S. Microbial biomass measurements in forest soils: Determination of $\mathrm{Kc}$ values and tests of hypotheses to explain the failure of the chloroform fumigation-incubation method in acid soils. Soil Biol. Biochem., 19:689-696, 1987b.

VANCE, E.D.; BROOKES, P.C. \& JENKINSON, D.S. Microbial biomass measurements in forest soils: The use of the chloroform fumigation-incubation method in strongly acid soils. Soil Biol. Biochem., 19:697-702, 1987c.

WARDLE, D.A. Controls of temporal variability of soil microbial biomass: A global-scale synthesis. Soil Biol. Biochem., 30:1627-1637, 1997.

WARDLE, D.A. \& GHANI, A. Why is the strength of relationships between pairs of methods for estimating soil microbial biomass often so variable? Soil Biol. Biochem., 27:821-828, 1995.

WARDLE, D.A. \& PARKINSON, D. Determination of bacterial and fungal fumigation $\mathrm{K}_{\mathrm{c}}$ - factors across a soil moisture gradient. Soil Biol. Biochem., 22:811-816, 1990a.

WARDLE, D.A. \& PARKINSON, D. Response of the soil microbial biomass to glucose, and selective inhibitors, across a soil moisture gradient. Soil Biol. Biochem., 22:825834, 1990b. 